\title{
Computing Extremal Quasiconformal Maps
}

\author{
Ofir Weber Ashish Myles Denis Zorin
}

Courant Institute of Mathematical Sciences, New York University, USA

\begin{abstract}
Conformal maps are widely used in geometry processing applications. They are smooth, preserve angles, and are locally injective by construction. However, conformal maps do not allow for boundary positions to be prescribed. A natural extension to the space of conformal maps is the richer space of quasiconformal maps of bounded conformal distortion. Extremal quasiconformal maps, that is, maps minimizing the maximal conformal distortion, have a number of appealing properties making them a suitable candidate for geometry processing tasks. Similarly to conformal maps, they are guaranteed to be locally bijective; unlike conformal maps however, extremal quasiconformal maps have sufficient flexibility to allow for solution of boundary value problems. Moreover, in practically relevant cases, these solutions are guaranteed to exist, are unique and have an explicit characterization. We present an algorithm for computing piecewise linear approximations of extremal quasiconformal maps for genus-zero surfaces with boundaries, based on Teichmüller's characterization of the dilatation of extremal maps using holomorphic quadratic differentials. We demonstrate that the algorithm closely approximates the maps when an explicit solution is available and exhibits good convergence properties for a variety of boundary conditions.
\end{abstract}

Categories and Subject Descriptors (according to ACM CCS): I.3.5 [Computer Graphics]: Computational Geometry and Object Modeling-Geometric algorithms, languages, and systems

Keywords: parametrization, quadrangulation, remeshing, conformal parametrization.

\section{Introduction}

Surface-to-plane and surface-to-surface maps arising in many geometry processing contexts (for example, texture mapping, remeshing and quadrangulation and surface registration) are expected to have a number of natural properties, such as geometric invariance, smoothness, local injectivity and mesh independence. These maps often have to satisfy a variety of boundary constraints, most commonly, positional constraints at interior and border points.

Consider a basic common problem of mapping a simply connected planar domain $D$ to another planar domain $D^{\prime}$, with fixed values on the boundary $f_{0}: \partial D \rightarrow \partial D^{\prime}$. In the case $D^{\prime}$ is convex, minimizing the Dirichlet energy yields a good solution: a harmonic map is unique, smooth and globally bijective. On the other hand, if $D^{\prime}$ is not convex, harmonic maps are no longer bijective and have fold singularities.

Remarkably, a different choice of energy functional yields maps that retain many aspects of harmonic maps for convex target domains, but does not require a convexity restriction. Extremal quasiconformal maps are maps minimizing the maximal deviation from conformality (dilatation, defined in Section 3). For fixed boundary data, the extremal map is unique, bijective (if the boundary allows this) and almost everywhere smooth (these maps may be only continuous at some isolated points).

While the functional is nonlinear and does not depend smoothly on the map, the solutions of the optimization problem, under weak assumptions, can be characterized by a single holomorphic function (a quadratic differential).

In this paper we present a numerical algorithm allowing to compute piecewise-linear approximations to extremal quasiconformal maps, using their characteristic properties. While the algorithm is nonlinear, we demonstrate that it converges reliably for a broad range of simply and multiply connected domain shapes and boundary data, can be naturally combined with other distortion optimization and is easy to implement.

\section{Related work}

The literature on surface parametrization is vast, and we refer to comprehensive surveys [FH05], [AS06] of earlier work in the area.

Comparison of functionals. We start with an overview of functionals commonly used for parametrization. The prob- 
O. Weber \& A. Myles \& D. Zorin / Computing Extremal Quasiconformal Maps

\begin{tabular}{|l|r|r|r|r|r|}
\hline \multicolumn{1}{|c|}{ functional/PDE } & LIN & BC & FLD & UNI & BIJ \\
\hline $\begin{array}{l}\text { harmonic } \\
\text { [Tut63, Flo97] } \\
\text { [DMA02, } \\
\text { LPRM02] }\end{array}$ & + & + & finite & + & convex \\
\hline $\begin{array}{l}\text { conformal } \\
\text { [GY03, KSS06, } \\
\text { BGB08, SSP08] }\end{array}$ & - & - & N/A & + & any \\
\hline $\begin{array}{l}\text { least-stretch } \\
\text { [SSGH01] }\end{array}$ & - & + & $\infty$ & - & any \\
\hline $\begin{array}{l}\text { MIPS } \\
\text { [HG99] }\end{array}$ & - & + & $\infty$ & - & any \\
\hline $\begin{array}{l}\text { elasticity } \\
\text { [Bal81, CLR04] }\end{array}$ & - & + & $\infty$ & - & any \\
\hline extremal q.c. & - & + & finite & + & any \\
\hline
\end{tabular}

Table 1: Properties of smooth parametrization functionals.

lem of defining a mapping from a surface to the plane or between surfaces has two principal aspects: a smooth definition (typically an energy measuring distortion) and corresponding discrete energy. The choice of underlying smooth energy is essential, as the flaws in this choice cannot be fully resolved by a good choice of discretization; in the limit of fine meshes the behaviors are likely to be similar.

Many energies and PDEs were proposed for parametrization and related problems. The most important ones are summarized in Table 1. We focus on several important properties, linearity of defining equations (LIN), whether mappings in this class can satisfy arbitrary boundary conditions (BC), whether the solution is unique (UNI) and for which domains it is bijective (BIJ).

An additional property, particularly important for nonlinear functionals, is their behavior for maps with folds (column FLD). We observe that all functionals ensuring bijectivity on arbitrary domains typically achieve this by fast growth to infinity as the mapping develops folds. As a consequence, the functionals are nonlinear, and require a starting point for optimization. This combination of properties results in a fundamental problem: a bijective map is needed to initialize the optimization, as the functional is infinite for any nonbijective initial map.

These observations highlight the unique properties of extremal quasiconformal maps: the functional remains finite on folds, and, under relatively weak assumptions on boundary data, has a unique minimizer.

MIPS parametrization introduced in [HG99] is of particular interest, as it is an integral of a symmetric form of a dilatation measure and is closely related to extremal quasiconformal maps, as discussed in Section 3.

Constrained parametrization. Methods for constrained parametrization were proposed in [Lév01, ESG01, KSG03, KGG05]. The method of [Lév01] optimizes Dirichlet energy and imposes pointwise position and gradient constraints with penalty terms, without attempting to guarantee bijectivity. Other techniques [ESG01, KSG03, KGG05] take an approach that can be characterized as achieving bijectivity first, possibly with mesh refinement, and without attempt-

ing to approximate a smooth functional, and then smoothing the resulting parametrization while enforcing the bijectivity constraint. The problem of this class of approaches is that the optimal constrained solution is likely to be on the boundary of the feasible domain, which negatively affects the parametrization quality.

Quasiconformal maps. The work most closely related to ours is the work on quasiconformal maps and Beltrami flows. In [ZRS05], quasi-harmonic maps are considered (harmonic maps in a user-specified metric). One can view these as approximations to quasi-conformal maps.

Quasiconformal maps, typically on disk domains, were computed by solving the Beltrami equation with prescribed Beltrami coefficient, e.g. in [Dar93]. [ZLYG09] proposes a method for computing quasiconformal maps from Beltrami coefficients on general multiply connected domains. In [LWT*10], holomorphic Beltrami flow is introduced for shape registration. Beltrami coefficient is used as a shape representation and two shapes are matched by evolving the Beltrami coefficient so that the landmarks are matched and the $L^{2}$ norm of the Beltrami coefficient, is minimized.

[LKF12] demonstrates that in the special case of four point constraints and periodicity conditions, an extremal quasiconformal map from the complex plane to itself has closed form.

In mathematics, the theory of Teichmüller maps and spaces has a long history, starting with the work of Grötzsch [Grö30], with key ideas introduced by Teichmüller [Tei40, Tei43] and it remains an active area of research. Many fundamental questions were settled by Ahlfors [Ahl53]. A number of books, e.g., [Ahl66, GL00] provide a comprehensive introduction to the subject.

Teichmüller spaces, i.e. spaces of conformal equivalence classes of surfaces, were introduced to graphics and vision literature in [SM04, JZLG09], and further developed in [JZDG09, WDC* 09 ]. The extremal dilatation of quasiconformal maps define a metric on these spaces, but this metric was not used in this work.

The interest in quasiconformal maps is to great extent motivated by the attractive properties of conformal maps, for which a number of high-quality computational algorithms were proposed [GY03, BGB08, KSS06, SSP08]. ABF [SdS01] can also be viewed as a conformal map approximation. Last three methods (when the optimization converge to a valid solution) guarantee bijectivity of discrete maps, these algorithms allow for free boundaries, or curvature constraints on the boundary, but not positional constraints. ARAP $\left[\mathrm{LZX}^{*} 08\right]$ minimizes deviation from isometry.

Global parametrization methods deal with surfaces of higher genus, and correspond to flat metrics with cones, e.g., recent feature aligned methods [RLL*06, KNP07, BZK09]. In the context of bijective maps, [BZK09] is of particular interest, proposing iterative stiffening as a way to eliminate foldovers inherent to harmonic-like parametrizations with constraints. 


\section{Background}

In our exposition we primarily focus on maps from plane to plane. Due to conformal invariance, all concepts can be easily generalized to surfaces of genus zero by mapping the surface conformally to the plane. However, as we briefly explain in Section 6 all concepts and the algorithm can be extended to surfaces of arbitrary genus.

We will use complex coordinates in the plane, $z=x+\mathrm{i} y$. For an arbitrary (not necessarily holomorphic) differentiable function $f(z)$, the complex derivatives are $f_{z}=\frac{1}{2}\left(f_{x}-\mathrm{i} f_{y}\right)$ and $f_{\bar{z}}=\frac{1}{2}\left(f_{x}+\mathrm{i} f_{y}\right)$. We assume that for all maps these derivatives are defined almost everywhere, and are squareintegrable. For each complex number $z$ we define a matrix $A(z)=\left(\begin{array}{rr}\operatorname{Re}(z) & -\operatorname{Im}(z) \\ \operatorname{Im}(z) & \operatorname{Re}(z)\end{array}\right)$ and a vector $v(w)=$ $(\operatorname{Re}(w), \operatorname{Im}(w))^{\top}$, so that $z w$ corresponds to $A(z) v(w)$.

Dilatation and quasiconformal maps. The little dilatation of a differentiable map $f$ at $z$ is defined as

$$
k(z)=\left|\frac{f_{\bar{z}}}{f_{z}}\right|
$$

It is related to the ratio $K$ of singular values of the Jacobian of $f$ (large dilatation) by $K=\frac{1+k}{1-k}$. A map $f: D \rightarrow D^{\prime}$ is quasiconformal if $k(z) \leq k$ for some $k$ almost everywhere in $D$. In this case, $k(f)=\sup k(z)$ is the maximal dilatation of the map. The dilatation $k$ has the following important properties:

- $0 \leq k<1$ for orientation-preserving maps, and $1 \leq k \leq \infty$ for orientation reversing maps.

- for a conformal map $k=0$ and a conformal map composed with reflection $k=\infty$.

An advantage of considering $k$ instead of $K$ is that $k$ varies continuously as the sign of the determinant of the Jacobian of $f$ changes on folds, while $K$ has a singularity (cf. comparison of functionals in Section (2)).

For a quasiconformal map with $f_{z} \neq 0$ almost everywhere, $\mu=f_{\bar{z}} / f_{z}$ is called the complex Beltrami coefficient, (Figure 1) and the equation

$$
f_{\bar{z}}=\mu f_{z}
$$

is the Beltrami equation.

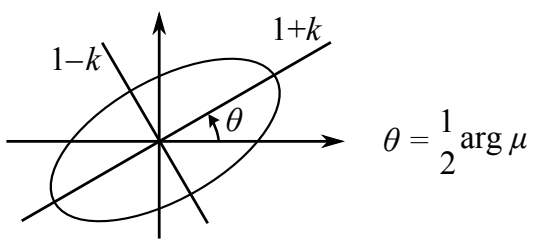

Figure 1: Geometric meaning of the complex Beltrami coefficient $\mu=k e^{2 i \theta}$.

If $g$ and $h$ are conformal, then $h \circ f \circ g$ is quasiconformal with the same dilatation $k$ as $f$. The simplest map with dilatation $k$ is an affine stretch $A_{k}$ along the two coordinate axes by $1+k$ and $1-k$ respectively; this yields a family of quasiconformal maps with constant dilatation $k(z)=k$, of the form

$$
f=h \circ A_{k} \circ g .
$$

This simple example of quasiconformal maps plays an important role in the theory of extremal quasiconformal maps.

Extremal quasiconformal maps. Suppose a quasiconformal map $h: D \rightarrow D^{\prime}$ maps a (possibly multiply connected) domain $D$ to $D^{\prime}$. Let $B$ be a subset of the boundary of $D$, and consider the set $Q_{B}$ of all quasiconformal maps $f$ that agree with $h$ on $B$ and are homotopic to $h$ (Figure 2). In the set $Q_{B}$ there is a map $f^{\text {ext }}$, with $k\left(f^{\text {ext }}\right) \leq k(f)$ for any $f$. The map $f^{\text {ext }}$ is called the extremal quasiconformal map.
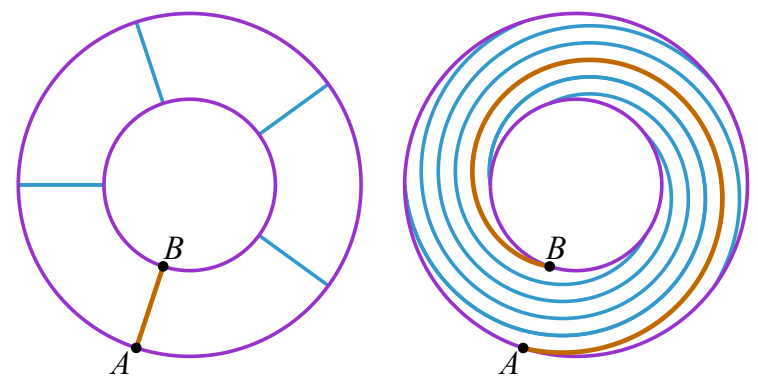

Figure 2: Examples of nonhomotopic maps with the same boundary data on ring domain; the left plot represents identity; note that the path $A B$ connecting two boundary points on the right, cannot be continuously deformed to the spiral path $A B$ on the left, while keeping the boundary fixed. An extremal quasiconformal map is unique for each homotopy class under assumptions of Proposition 2.
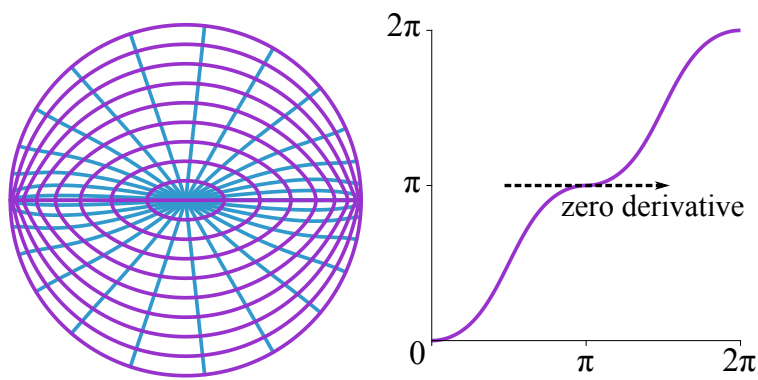

Figure 3: Left: A non-Teichmüller extremal map of the disk to itself. The image of the polar coordinate grid is shown. Right: the boundary values of the map on the circle in angular coordinates.

For a broad range of practically relevant boundary conditions, extremal quasiconformal maps have a very specific local structure that can be described in terms of a pair of conformal maps, and a global structure that can be characterized by a quadratic differential. 
Teichmüller maps. Locally in a neighborhood of every point (excluding zeros of $g_{z}$ ), a Teichmüller map has the form (3): $h \circ A_{k} \circ g$, for a choice of conformal maps $h(w)$ and $g(z)$, where $w$ denotes the complex coordinate on the intermediate domain $A_{k}$ acts on.

A simple calculation leads to the following lemma:

Lemma 1 For a map $f=h \circ A_{k} \circ g$, where $h$ and $g$ are conformal, and $A_{k}$ is a stretch with dilatation $k$, the Beltrami coefficient has the form

$$
\frac{f_{\bar{z}}}{f_{z}}=k \frac{\bar{\phi}}{|\phi|}
$$

where $\phi=\left(g_{z}\right)^{2}$.

Proof By Cauchy-Riemann equations, $h_{\bar{w}}=0$, and $g_{\bar{z}}=0$; similarly, for the anti-conformal map $\bar{g}, \bar{g}_{z}=0$. Observe that the affine map $A_{k}(w)$ in complex form can be written as $A_{k}(w)=w+k \bar{w}$. For this reason, $f_{z}=h_{w}\left(g_{z}+k \bar{g}_{z}\right)=h_{w} g_{z}$, and $f_{\bar{z}}=h_{w}\left(g_{\bar{z}}+k \bar{g}_{\bar{z}}\right)=k h_{w} \bar{g}_{\bar{z}}$ (because $g$ is conformal, $g_{\bar{z}}=$ 0 and $\left.\bar{g}_{z}=0\right)$. We observe that $f_{\bar{z}} / f_{z}=k \bar{g}_{\bar{z}} / g_{z}=k \bar{g}_{\bar{z}}^{2} /\left|g_{z}^{2}\right|$, as $\bar{g}_{\bar{z}}=\overline{g_{z}}$.

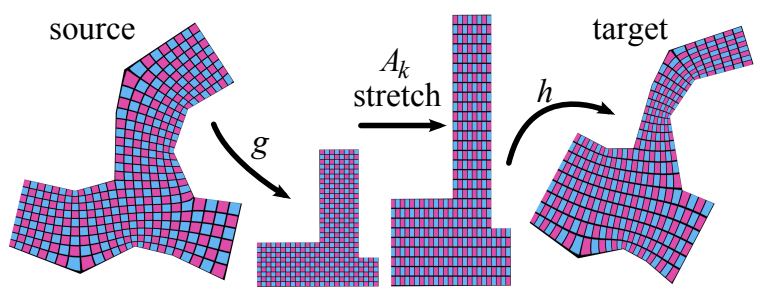

Figure 4: Locally, a Teichmüller map can be decomposed into two conformal maps and an affine transform.

The quantity $\phi=g_{z}^{2}$ is called the quadratic differential of $f$. Geometrically, the quadratic differential can be interpreted, up to a scale, as the traceless part of the metric tensor $M=J[f]^{\top} J[f]$ of the map $f$, where $J[f]$ is the Jacobian matrix. More specifically, the singular vectors of $A(\phi(z))$ determine the stretch directions of $f$ at $z$.

More generally, a quadratic differential on a domain with a complex coordinate $z$ is defined by a holomorphic function $\phi(z)$, which depends on the choice of the coordinate system. If $w$ is a different choice of complex coordinates on the domain, then $\phi^{w}(w) w_{z}^{2}=\phi(z)$. This change-of-coordinates rule also allows to define a quadratic differential on a Riemann surface by a set of holomorphic functions on local charts.

Definition 1 On a planar domain $D$, a map $f$ is called a Teichmüller map, if it is quasiconformal with Beltrami coefficient of the form

$$
\mu(z)=k \frac{\bar{\phi}(z)}{|\phi(z)|}
$$

where $\phi$ is a holomorphic function and $k$ is a constant dilatation, everywhere where $\phi \neq 0$.
Observe that the definition is invariant with respect to composition with conformal maps on either side. While postcomposition does not change the Beltrami coefficient, precomposition results in a change in $\phi$ but not in $k$.

Unlike the definition of extremal quasiconformal maps, involving minimization of a nonsmooth and nonlinear functional, Teichmüller maps are much more amenable to computation, thanks to explicit form of the Beltrami coefficient. Fortunately, in most cases, an extremal map is a Teichmüller map and it is unique. In particular, the following proposition can be obtained from a sufficient condition for Teichmüller map existence for disks [Rei02] and conformal mapping properties:

Proposition 2 For two simply connected bounded domains $D$ and $D^{\prime}$ with piecewise smooth boundaries forming nondegenerate corners; suppose $h: \partial D \rightarrow \partial D^{\prime}$ is a function with continuous nonvanishing bounded derivative and almost everywhere defined second derivative. Then in every homotopy class of maps $f$ satisfying $\left.f\right|_{\partial D}=h$, there is a unique extremal Teichmüller map $f$ with bounded $L_{1}$-norm of the quadratic differential $\phi$.

As illustrated in Figure 3, the conditions on $h$ in proposition 2 are essential. The quasiconformal map of the disk to itself shown in this image is known to be extremal for its boundary values [Rei02]. The map in the image is obtained as $f_{2} \circ g \circ f_{1}$, where $f_{1}(z)=\mathrm{i}(1+z) /(1-z)$ maps the disk to the upper half-plane, $f_{2}$ is its inverse, and $g(z)=|z| z$, and can be easily shown not to be a Teichmüller map.

A similar statement appears to hold for multiply connected domains [Gar], but we were unable to locate a published proof. These theorems extend immediately to surfaces of genus zero with boundary, and also hold for maps between surfaces of arbitrary finite genus. In this paper, we focus on genus zero.

Proposition 2 is the foundation of our approach for the computation of extremal maps. For these types of boundary data, it is sufficient to find a map with holomorphic quadratic differential and constant dilatation.

Quadratic differential structure. Before discussing the algorithm we briefly mention the local structure of quadratic differentials near its zeros and poles. The quadratic differentials of Teichmüller maps have only simple poles, and generically zeros of order 1 . The trajectories of quadratic differentials are integral lines of the fields of singular directions of $A(\phi(z))$. These directions change rapidly near zeros and poles, as can be seen in Figure 5. While poles of quadratic differentials always remain on the boundaries, zeros spontaneously appear in the interior or on the boundary (Figure 9).

\section{Algorithm}

Overview. Given a boundary function $f_{b}$ and an initial (not necessarily bijective) map satisfying boundary conditions, we compute a piecewise-linear approximation to a Teichmüller map minimizing a discretization of the least-squares Beltrami (LSB) energy:

$$
E_{L S B}=\int_{D}\left|f_{\bar{z}}-k \frac{\bar{\phi}}{|\phi|} f_{z}\right|^{2} d A
$$



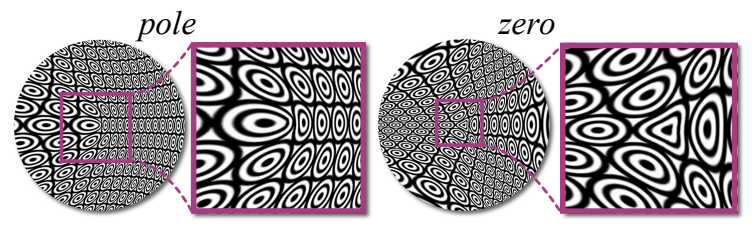

Figure 5: Behavior of a Teichmüller map near poles and zeros of a quadratic differential.

with $f, \phi$ and $k$ are unknowns, and the constraints $\phi_{\bar{z}}=0$ (i.e. $\phi$ is holomorphic) and $\left.f\right|_{\partial D}=f_{b}$ are applied.

By Teichmüller uniqueness and existence, this energy has a single global minimum with value zero, in the homotopy class of its initial point. We note that the energy is not convex. However, we have observed (see Section 5) that it consistently converges starting from a variety of arbitrary initial starting points.

There are two essential conditions defining the Teichmüller map that need to be converted to a discrete form: (1) the quadratic differential defining the map has to be holomorphic (2) the dilatation $k$ has to be constant. Note however that a discrete piecewise-linear map cannot, in general, achieve a perfectly constant $k$, and as a consequence, zero LSB energy. This is also the case for discrete conformal maps, for which $k$ should be constant zero, but deviates from zero significantly (this is demonstrated in Figure 6).

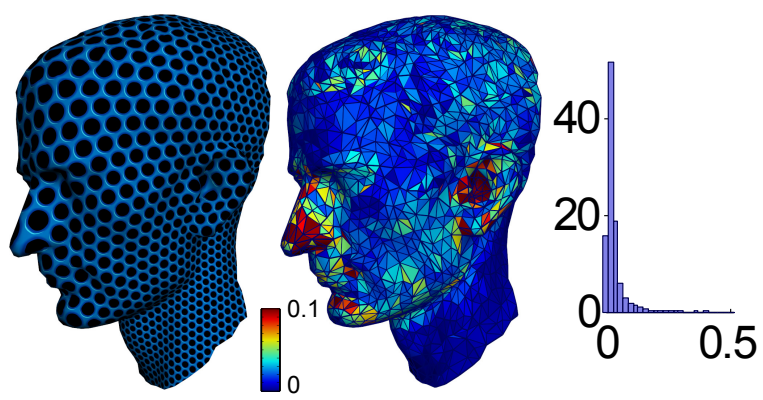

Figure 6: The distribution of dilatation values for a conformal map computed using the method of [SSP08]. Note that although the method produces a high-quality conformal map, the dilatations on triangles may be far from zero.

\subsection{Discretization}

We represent $f$ as a piecewise-linear map defined by values at vertices of the original mesh. The complex derivatives $f_{\bar{z}}$ and $f_{z}$ are naturally defined per triangle. If $\mathbf{e}_{i}, \mathbf{e}_{j}, \mathbf{e}_{k}$, are edge vectors of a triangle $T$ with opposite vertices $(i, j, k)$, the gradient of piecewise-linear $f$ is given by $\frac{f_{i} \mathbf{t}_{i}+f_{j} \mathbf{t}_{j}+f_{k} \mathbf{t}_{k}}{2 A_{T}}$, where $A_{T}$ is the triangle area, $\mathbf{t}_{i}=\mathbf{e}_{i}^{\perp}$, and $f_{i}$ are values at the vertices. It immediately follows that per-triangle derivatives are given by

$$
\begin{aligned}
& f_{z}=\frac{1}{4 A_{T}}\left(f_{i} \bar{t}_{i}+f_{j} \bar{t}_{j}+f_{k} \bar{t}_{k}\right), \\
& f_{\bar{z}}=\frac{1}{4 A_{T}}\left(f_{i} t_{i}+f_{j} t_{j}+f_{k} t_{k}\right)
\end{aligned}
$$

where $t_{i}=t_{i}^{x}+\mathrm{i} t_{i}^{y}$ is the complex form of the vectors $\mathbf{t}_{i}=$ $\left(t_{i}^{x}, t_{i}^{y}\right)$.

Discretization of quadratic differentials. The holomorphic condition on the quadratic differential $\phi, \phi_{\bar{z}}=0$ can be discretized in a similar manner if the quadratic differential values are defined per vertex. However, this definition does not naturally reflect the relation between $f$ and $\phi$. Equation (2) suggests that the Beltrami coefficient $\mu$, and, as a consequence, the quadratic differential $\phi$, is most naturally defined in a way consistent with $f_{\bar{z}}$ and $f_{z}$, i.e., per triangle. If we use notation $\bar{\phi}=v_{x}+\mathrm{i} v_{y}$ for the components of the conjugate of the quadratic differential, then the holomorphic condition $\phi_{\bar{z}}=0$ can be written more explicitly as $\partial_{x} v_{x}+\partial_{y} v_{y}=0$ and $\partial_{y} v_{x}-\partial_{y} v_{y}=0$, with two equations corresponding to equating real and imaginary parts of $\phi_{\bar{z}}$ to zero.

If we regard the pair $\left(v_{x}, v_{y}\right)$ as components of a 1-form $h=v_{x} d x+v_{y} d y$, then $d h=\left(\partial_{y} v_{x}-\partial_{x} v_{y}\right) d x \wedge d y$. Similarly, as the Hodge star acts on 2D 1-form components as a 90degree rotation, we get for the co-boundary operator $\delta h=$ $* d * h=\partial_{x} v_{x}+\partial_{y} v_{y}$. Thus, we conclude that the 1-form $h=$ $\operatorname{Re}(\phi) d x-\operatorname{Im}(\phi) d y$ is harmonic:

$$
d h=0, \quad \delta h=0 .
$$

If we regard $\mathbf{v}=\left(v_{x}, v_{y}\right)$ as a vector field, this pair of equations correspond to zero curl and divergence of $\mathbf{v}$. This observation suggests a standard per-edge discretization, with real scalars associated with edges, and standard discretizations of $d$ and $\delta$ operators. Let $h_{i j}$ be the value of the harmonic 1 -form on the edge $e_{i j}$. For convenience, we use notation $h_{j i}=-h_{i j}$.

For a triangle $T=(i, j, k)$, let $\mathbf{r}_{i j}=\mathbf{e}_{j}^{\perp}-\mathbf{e}_{i}^{\perp}$, and define $\mathbf{r}_{j k}$ and $\mathbf{r}_{k i}$ by cyclic permutation. We represent the vectors $\mathbf{r}=\left(r_{x}, r_{y}\right)$ in complex form $r=r_{x}+\mathrm{i} r_{y}$.

Then the per-triangle quadratic differential values are defined by

$$
\phi_{T}=\frac{1}{6 A_{T}}\left(h_{i j} r_{i j}+h_{j k} r_{j k}+h_{k i} r_{k i}\right)
$$

Discrete energy and constraints. To summarize, the energy (5) is discretized using per-vertex complex variables $f_{i}$ to represent the map, a single scalar $k$ for the constant dilatation and a real harmonic vector 1-form represented by per-edge values $h_{i j}$. The energy is given by

$$
E_{L S B}^{d}=\sum_{T_{m}}\left|f_{\bar{z}}^{m}-k \frac{\bar{\phi}_{m}}{\left|\phi_{m}\right|} f_{z}^{m}\right|^{2} A_{m}
$$


with $\phi$ defined by (8), and $f_{z}$ and $f_{\bar{z}}$ defined by (6). Additional constraints ensure that $h$ is harmonic:

$$
\begin{array}{r}
h_{i j}+h_{j k}+h_{k i}=0, \text { for triangle } T=(i, j, k) \\
\sum_{j} w_{i j} h_{i j}=0, \text { for a vertex } j,
\end{array}
$$

where $w_{i j}$ are the standard cotangent weights, with the two conditions corresponding to vanishing boundary and coboundary operators $d$ and $\delta$.

We introduce an auxiliary variable $s_{m}=1 /\left|\phi_{m}\right|$. With this variable, the energy takes the form

$$
E_{L S B}^{d}=\sum_{T_{m}}\left|f_{\bar{z}}^{m}-k \bar{\phi}_{m} s_{m} f_{z}^{m}\right|^{2} A_{m}
$$

with an additional nonlinear constraint $s_{m}=1 /\left|\phi_{m}\right|$. While we focus on Dirichlet boundary conditions defined on the whole boundary, we note that free boundaries can be easily added.

\subsection{Optimization}

The energy (9) is highly nonlinear. Standard methods for optimizing this energy that we have tried (quasi-Newton methods) do not behave well (see Section 5). At the same time, the energy is quadratic with respect to each of the vector variables $\mathbf{f}=\left[f_{1}, \ldots f_{P}\right]$ and $\mathbf{h}=\left[h_{1}, \ldots h_{L}\right]$ where $P$ and $L$ are the number of vertices and edges respectively. It is also quadratic with respect to the global variable $k$. The nonlinearity in the solution reduces to enforcing the constraint between $s_{m}$ and $\phi_{m}$.

This observation suggests an alternating-descent algorithm, with $\mathbf{h}$, $\mathbf{f}$ and $k$ minimized in alternating fashion. While this does not guarantee convergence to a global minimum, this yields a stable algorithm with consistent practical behavior (See Section 5). If $\mathbf{f}, \mathbf{h}$ are fixed, $k$ can be easily computed explicitly. Furthermore, for fixed $\mathbf{f}$, we use the following property of the energy:

Proposition 3 For fixed f, optimal discrete holomorphic $\phi$ (up to a scale factor) does not depend on $k$, and optimal $k$ is given by

$$
k=\sum_{m} 2 A_{m} \frac{\operatorname{Re}\left(\hat{\mu}_{m} \overline{f_{\bar{z}}^{m}}\right)}{\sum_{m} A_{m}\left|f_{\bar{z}}^{m}\right|^{2}}
$$

where $\hat{\mu}_{m}=\frac{\bar{\phi}_{m}}{\left|\phi_{m}\right|}$. This formula is easily obtained by direct computation.

The algorithm in its basic form (Algorithm 1) alternates two steps. We use a harmonic $\mathbf{f}$ to initialize the iterative solution, corresponding to minimizing LSB energy with constant $k=0$. In the main loop, a quadratic problem is solved to determine an optimal value $\mathbf{h}^{\text {new }}$, keeping $\mathbf{f}$ and $\mathbf{s}$ fixed, in general violating the nonlinear constraint $s_{m}=1 /\left|\phi_{m}\right|$. The constraint is enforced by projection, and a line search is performed in the direction determined by $\mathbf{h}^{\text {new }}$, to ensure that the energy decreases.

The stability of this algorithm is guaranteed as the energy is forced to decrease at each step; boundary conditions are also satisfied by construction. Its main limitation is that

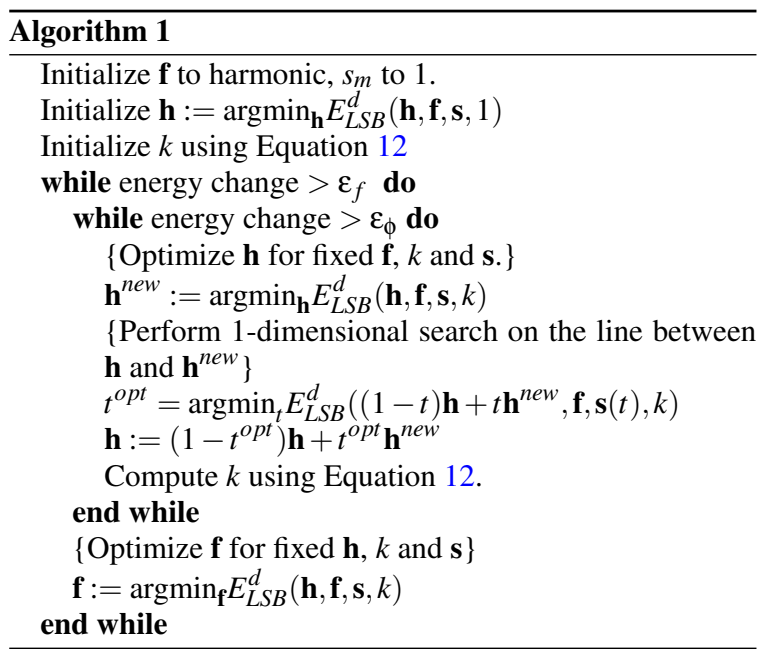

there is no guarantee that the global minimum is reached or approximated well. In practice, we observe that the algorithm converges to the same solution, even for large variations in the starting point; furthermore, in all our test cases for which the global minimum is known, the algorithm converges to this global minimum, as discussed in more detail in Section 5 .

Extension to surfaces. The algorithm directly extends to mapping genus-zero surfaces with boundary to the plane. Because quasiconformal distortion is conformally invariant, it is sufficient to use an arbitrary conformal map of the surface to the plane, and the composition of this conformal map with the computed extremal quasiconformal map is extremal. We use the scale-factor based conformal map computation to map surfaces to the plane [BGB08, SSP08]. Specifically, for a multiply connected domain of genus zero we follow these steps:

- One boundary is chosen as external; the remaining boundary loops are triangulated, reducing the surface to simply connected.

- The algorithm of [SSP08] is used to compute a logarithmic scale factor $u_{i}$ for each vertex $u_{i}$, such that edge lengths $l_{i j}=e^{\left(u_{i}+u_{j}\right) / 2}$ define a planar mesh. For the solution to be unique, we choose the scale factors to be zero on the outer boundary (i.e., no scale distortion along the boundary).

- The added triangles are removed to obtain the discrete conformal image of the original mesh.

We note that the metric used for the conformal map need not coincide with the Euclidean embedding metric. This can be used for minimizing different types of errors in the parametrization (cf. [KMZ10]), as well as "correction" of a non-bijective parametrization (i.e. nearly flat metric). If the metric is derived from a different locally bijective parametrization, there is no need for the flattening conformal map; however if the parametrization is not locally bijective, the conformal map is still needed. Figure 18 shows some examples of this. 
Discussion. By construction, the quadratic differential computed by the algorithm is discrete holomorphic and the corresponding Beltrami coefficient has constant dilatation $k$. At the same time, the actual Beltrami coefficient of the map $f_{\bar{z}} / f_{z}$ is just an approximation to the "perfect" computed coefficient. We note that in general, if $\hat{\mu}$ (i.e. the angular part of $\mu$ ) is fixed, this determines the parametrization uniquely with no control for $k$ on each triangle.

While we observe good convergence of the algorithm to constant $k$ in $L^{2}$ norm when the mesh is refined, this does not guarantee that $k<1$ (i.e. there are no fold-overs) on all triangles, even for a very fine mesh. In practice, we observe that no triangles are flipped for a sufficiently fine mesh, excluding few triangles near highly concave parts of the boundary.

In the next section, we present a detailed evaluation of the behavior of the algorithm: dependency on the starting point, convergence rates, comparison to analytic cases.

\section{Evaluation}

Validation. We validate our method in several ways. The most direct validation is comparison to analytically computed maps. Unfortunately, the analytic solution is known explicitly only in few cases. We consider two maps known analytically: mapping a disk to itself with four boundary points moved to different locations, and mapping of a ring to a ring with a different ratio of inner to outer radius. In the first case, we consider maps moving points at angular locations $\pm \pi / 4, \pi \pm \pi / 4$ on a circle, to $\pm \phi, \pi \pm \phi$. This map is given by

$$
f_{\phi}(z)=e^{-i \phi} F\left(z e^{i \phi}, e^{-2 i \phi}\right)
$$

where $F(z, w)$ is the incomplete elliptic integral of the first kind. For the ring domain with inner and outer radii $r_{0}$ and $r_{1}$, mapped to a ring with inner and outer radii $r_{0}^{\prime}$ and $r_{1}^{\prime}$, the extremal map has the polar coordinate form

$$
(r, \phi) \rightarrow\left(r_{0}^{\prime}\left(r / r_{0}\right)^{K}, \phi\right)
$$

where $K=\ln \left(r_{1}^{\prime} / r_{0}^{\prime}\right) / \ln \left(r_{1} / r_{0}\right)$. In both cases, we observe an accurate match both for the maps, and extremal dilatation values.

We can also compute a subclass of Teichmüller maps semi-analytically, by prescribing a pair of conformal maps, and a stretch in the intermediate domain. We tested our method for both analytically and semi-analytically computed maps (Figure 7).

In analytic cases, the maps are very close to exact ones. As the piecewise linear maps cannot in general have constant dilatation, we consider the deviation from the correct constant $k$ as a measure of error, shown in the figure.

Convergence in the general case. While the optimal dilatation cannot be computed for general boundary conditions, one can estimate the rate at which the deviation of dilatation $k$ from the average value decreases. We observe a similar behavior for this measure shown in the last two examples in Figure 7. The number of iterations needed to reach $1 \%$ relative change in energy was typically on the order of 10
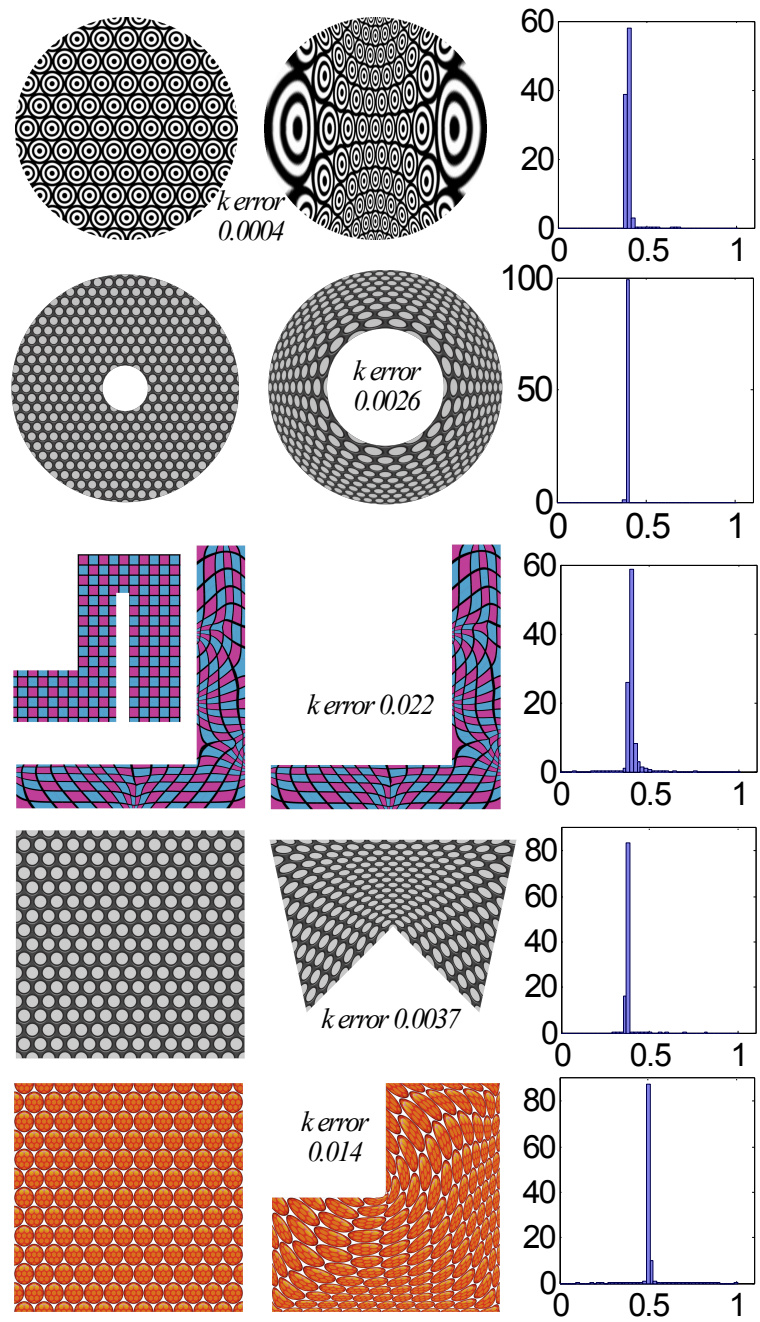

Figure 7: Top 3 examples: comparison to extremal quasiconformal maps. A disk with four points moved along the boundary, a ring with a change in the ratio of inner-to-outer radius, and an example of a map computed as a composition (3). This example shows a comparison of the map directly computed as the composition (left) with the map computed using our algorithm (middle). For each example, a histogram of $k$ is shown, with percentages of triangles along the vertical axis. The last two examples use prescribed boundary values not corresponding to a known map.

(in some cases, such as the ring domain, as low as 2-3). On the other hand, more complex and extreme deformations required more iterations.

As expected, we observe that zeros of the quadratic differential become visible in the map (e.g., as shown in Figure 9).

Dependence on initialization. As our algorithm minimizes a nonlinear energy, in principle the result may depend on the starting point. However, we observe very little dependence, as demonstrated in Figure 10. 
O. Weber \& A. Myles \& D. Zorin / Computing Extremal Quasiconformal Maps
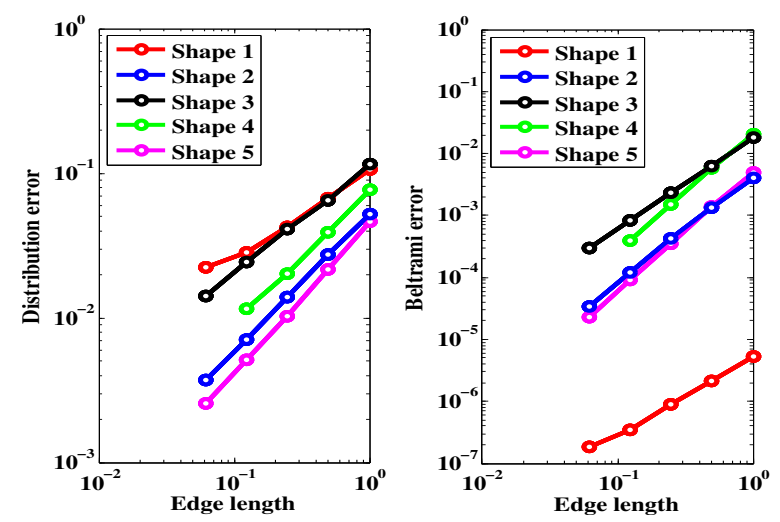

Figure 8: Convergence of $k$ as a function of mesh resolution for examples of Figure 7; for the ring and four-point disk examples, new meshes were generated for each resolution; for remaining examples, the meshes were refined by quadrisection.

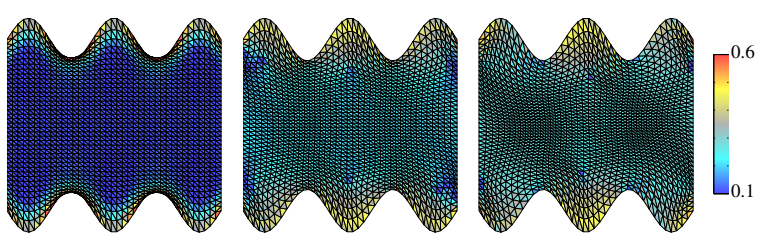

Figure 9: After 3 iterations of the algorithm, applied to the initial harmonic map, zeros of the quadratic differential become visible; in the final map they appear as isolated spots of nearly zero $k$.

Alternative methods for computing the map. As the extremal quasiconformal maps are defined as minima of the functional $\max _{z} k(z)$, an obvious alternative is to attempt to compute the map directly, without relying on its description as the Teichmüller map. The discrete problem can be formulated as a the problem of minimizing $k$ with per-triangle nonlinear inequality constraints $k_{m}(\mathbf{f})<k$. Our experiments show that with most starting points standard interior-point or active-set methods do not make much progress on the problem even in relatively simple cases (Figure 11, matlab optimization with analytic gradients is used). A common approach to approximation solutions of $L_{\infty}$ optimization problems is to use $L_{p}$ norms with increasing $p$. For low $p$, and high $p$ we have observed bad convergence behavior not producing meaningful maps. Medium-range values of $p(p=5)$ converged (although more than an order of magnitude slower than our method) but the solution was quite far from the extremal map.

Comparison with other energies. Figure 15 shows how our maps compare to several common types of maps obtained by minimizing other types of energies, that can satisfy Dirichlet boundary conditions: harmonic/LSCM ( [DMA02, LPRM02]), ARAP ( [LZX*08]), and MIPS [HG99]. The

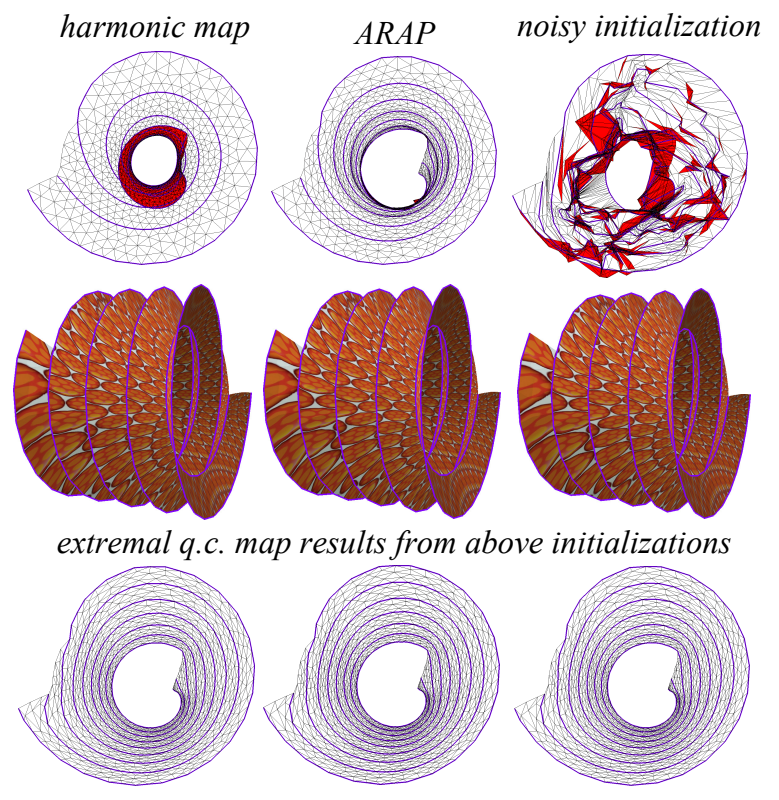

Figure 10: Dependence on initialization. A screw is mapped to an annulus using three different initializations. Helical sharp edges are highlighted to show parametrization flow. Upper row: Harmonic, ARAP and noisy parametrizations exhibit 924, 268 and 831 fold-overs respectively (shown in red). Lower two rows: Extremal q.c. maps resolve all foldovers. The algorithm produces consistent results for each initialization in the first row.
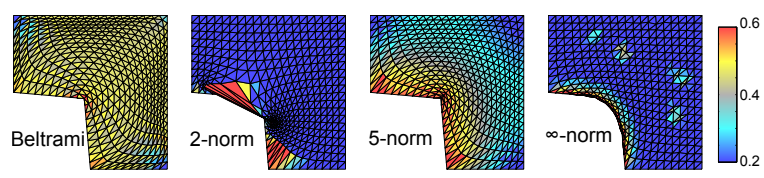

Figure 11: Comparison of our method with direct optimization of maximal $k$, and optimization of $\int(k)^{p} d A$ for 3 values of $p$. The color indicates the value of $k$.

first two methods could handle the same boundary constraints, but produced parametrizations far from bijective. MIPS had to be initialized with simpler boundary constraints, for which an initial bijective parametrization can be obtained. Figures 12 and 13 demonstrate challenging parametrizations with sharp feature alignments. Figure 14 compares our method to ARAP when applied to deform an image.

We observe that for our method the final result for higher resolution meshes is largely independent of mesh connectivity(Figure 16). For simple boundary shapes, the map is typically well-approximated with relatively few triangles (Figure 17).

Combining with other error metrics. While maximal dilatation are a natural measure of distortion, it does not cap- 


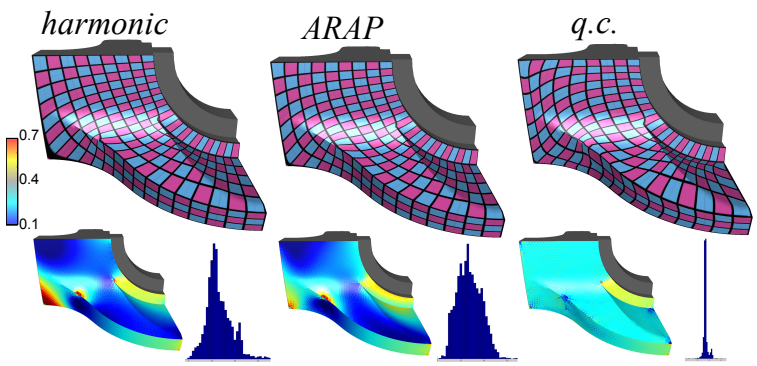

Figure 12: Parametrization with aligned sharp features and boundary. We cut the front of the fandisk model to obtain a disk-like surface and flatten it while aligning the sharp features and boundary to parametric lines using harmonic map, ARAP and our extremal q.c. map. The color visualization and histograms shows values of $k$ between 0.1 to 0.7.

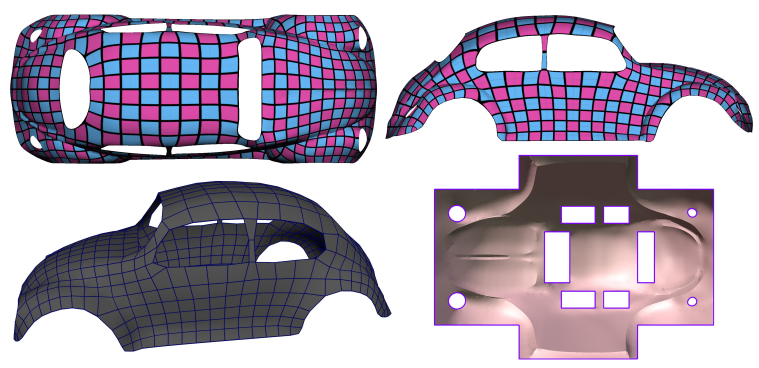

Figure 13: Parametrization aligned to the boundaries of a large curved multiply connected model using our q.c. extremal algorithm. The disk-topology of the surface is maintained. i.e., no cones or cuts are introduced. Top row: a texture of regular grid is mapped onto the surface. Bottom row: a quadrangular mesh (left) is obtained by sampling the parametric domain (right). In contrast to Figure 18, here we use the original surface metric.

ture many common types of requirements needed in applications. For example, the parametrization may need to be close to isometric, and dilatation does not take area distortion into account. In other cases, we may want the parametric lines to be aligned with features etc. One can use extremal quasiconformal maps in composition with a metric defined using a different parametrization type as explained in Section 4.2. Figure 18 shows several examples of this type.

Figure 19 demonstrates the behavior of our algorithm when pushed to the limit. A multiply connected ball shaped surface with twelve pentagonal holes is mapped to a disk without introducing any cuts. One of the holes is mapped to the boundary of the disk while all the others are mapped to fixed circles inside the disk. With such extreme boundary conditions, large amount of conformal distortion is unavoidable. Nevertheless our algorithm manage to produce a bijective map without any foldovers. Harmonic map with exact same boundary conditions is much smoother. However the

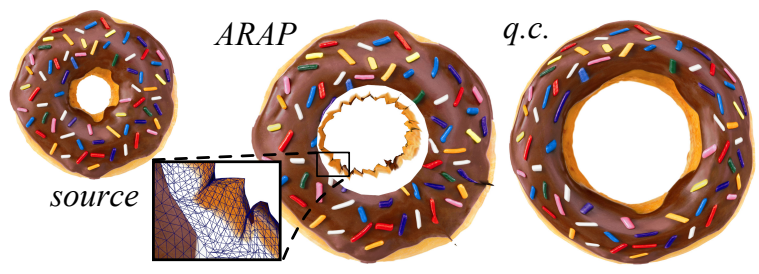

Figure 14: Image deformation. A doughnut is deformed to have different thickness using ARAP and extremal q.c. map. Note the foldovers ARAP develops.
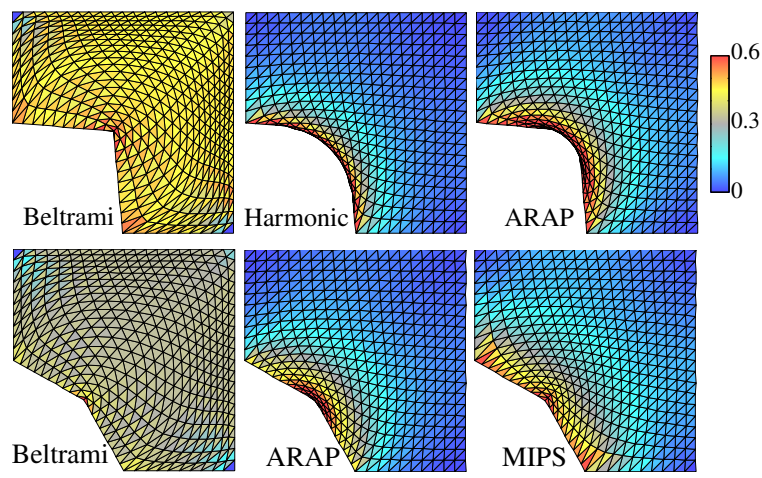

Figure 15: Comparison of the results for several energies: an extremal quasiconformal map, harmonic, ARAP, MIPS. As MIPS energy is infinite at foldovers, it was initialized on a less challenging configuration with bijective output of ARAP.

conformal distortion is extremely high, leading to more than 800 flipped triangles. ARAP parametrization produce similar result with more than 600 flipped triangles.

\section{Conclusions and future work}

From computational point of view, quasiconformal maps, i.e. maps with globally bounded dilatation, are a natural way

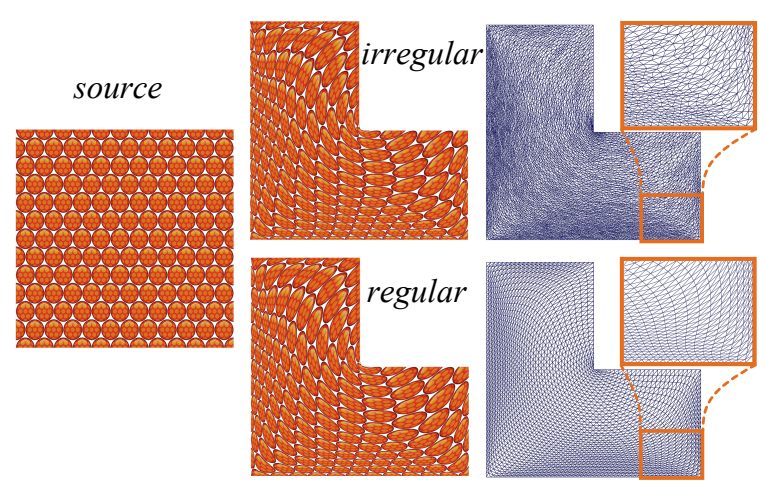

Figure 16: Comparison of maps obtained for regular and irregular meshes 


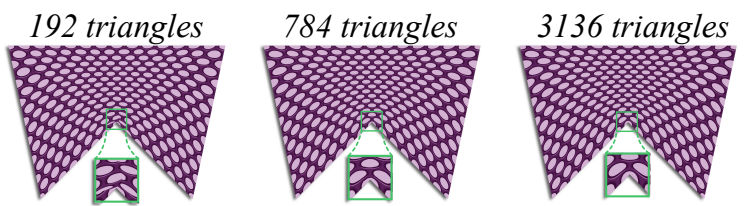

Figure 17: A sequence of maps obtained by mapping a square to a wedge shape; at each resolution, an irregular mesh is used.
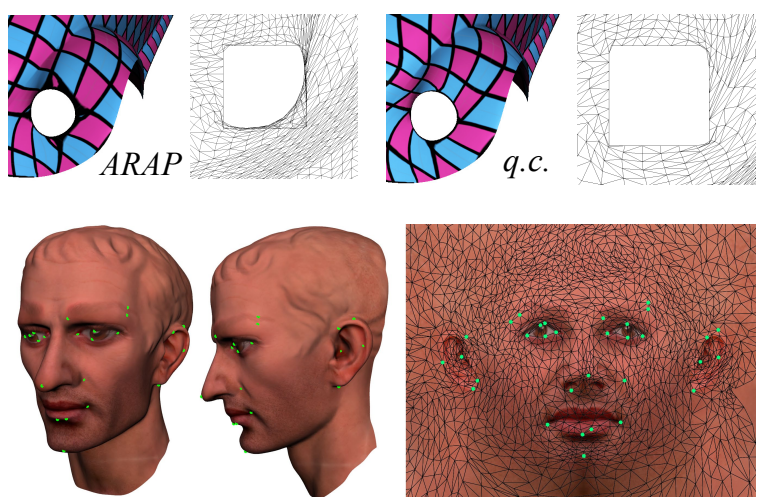

Figure 18:

Combining extremal quasi-conformal parametrization with other metric. Top, left: ARAP parametrization; Right: q.c. parametrization in ARAP metric reduces artifacts near constraints at the cost of increased distortion elsewhere. Bottom: q.c. parametrization in ARAP metric is used to move markers to desired positions.

to describe bijective maps, because for practical purposes, arbitrary high dilatation is as bad as the lack of bijectivity. This class of maps is almost as broad as all bijective maps, and considering extremal maps makes it possible to define a unique quasiconformal solution for problems with fixed boundaries. As we have demonstrated, although the problem is nonlinear, extremal maps can be approximated efficiently
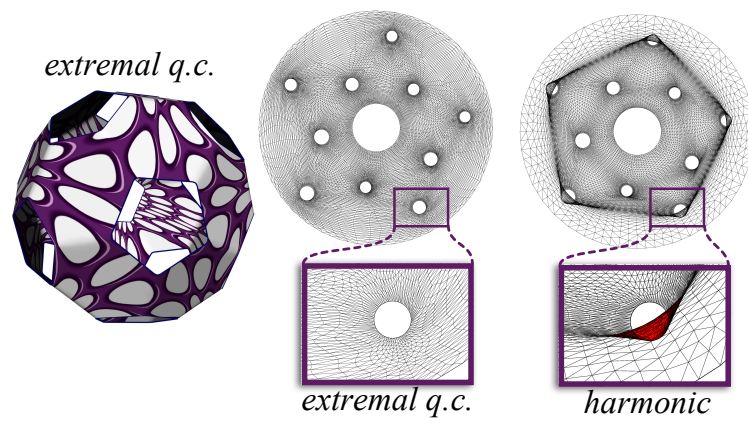

Figure 19: A ball is mapped to a disk with circular holes. One of the pentagonal holes on the ball is mapped to the outer boundary of a disk. A harmonic map results in a map with more than 800 flipped triangles. and robustly. At the same time, our approximation does not guarantee that the resulting approximate map is locally bijective everywhere.

We view developing a truly discrete concept of extremal quasiconformal maps as a promising direction. The generalization is far from trivial, as simply defining the extremal map as the global minimum of maximal per-triangle dilatation functional does not retain most attractive properties of the smooth quasiconformal maps.

Extension to surfaces of arbitrary genus and surfaces with cones. An important limitation of the described method is its inability to handle surfaces of arbitrary genus, and target domains with cones (cf. [SSP08, BGB08, BZK09]). We briefly outline how these cases can be handled, leaving a detailed specification of the algorithm and its practical evaluation as future work.

The overall approach is to use local simply connected charts to define the map. In fact, a single chart, obtaining by cutting the surface to a disk is sufficient, but the reasoning is easier to explain with multiple overlapping charts. Suppose the surface is covered by such charts.

Recall that the algorithm involves interleaved computation of a parametrization $f(z)$ and the quadratic differential $\phi(z)$. We can define these locally, using a conformal atlas: for each chart a conformal map to the plane is defined, assigning to each point $p$ its coordinate $z(p)$. We assume that these conformal maps are consistent, i.e. for two overlapping charts, with coordinates $z(p)$ and $z^{\prime}(p)$, in the overlap area the composition of conformal coordinates $w \circ z^{-1}=\chi$ is a conformal map.

We can now define a parametrization $f^{z}$ and a quadratic differential $\phi^{z}$ for each chart, requiring consistency. The consistency for the maps $f^{z}$ can be imposed in the usual way, as it is done in, e.g., [SSP08]: the maps $f^{z}$ and $f^{w}$ coincide, up to a rigid translation and rotation by an angle $k \pi / 2$, i.e. $f^{w}=e^{\mathrm{i} k \pi / 2} f^{z}$. For quadratic differentials the transformation rule, (see Section 3) is $\phi^{w}(w) \chi_{z}^{2}=\phi^{z}(z)$. As the quadratic differential is related to the metric tensor of $f$, the rigid transform between $f^{z}$ and $f^{w}$ values does not affect it.

As a result, we can obtain a similar algorithm for each chart, with additional constraints imposed on maps and quadratic differentials between charts.

\section{Acknowledgements}

We would like to thank Frederick Gardiner for helpful discussions. This research was partially supported by NSF awards IIS-0905502, DMS-0602235 and OSI-1047932.

\section{References}

[Ahl53] AHLFors L.: On quasiconformal mappings. Journal d'Analyse Mathématique 3, 1 (1953), 1-58. 2

[Ahl66] AHLFORS L.: Lectures on quasiconformal mappings, vol. 38. Amer. Mathematical Society, 1966. 2

[AS06] A. SHEFFER E. Praun K. R.: Mesh parameterization methods and their applications. Foundations and Trends in Computer Graphics and Vision 2, 2 (2006). 1 
[Bal81] BALL J.: Global invertibility of Sobolev functions and the interpenetration of matter. Proc. Roy. Soc. Edinburgh Sect. A 88, 3-4 (1981), 315-328. 2

[BGB08] Ben-Chen M., Gotsman C., Bunin G.: Conformal flattening by curvature prescription and metric scaling. Computer Graphics Forum 27, 2 (2008), 449-458. 2, 6, 10

[BZK09] Bommes D., Zimmer H., Kobbelt L.: Mixedinteger quadrangulation. ACM Trans. Graph. 28, 3 (2009), 77. 2,10

[CLR04] Clarenz U., Litke N., RumpF M.: Axioms and variational problems in surface parameterization. Computer Aided Geometric Design 21, 8 (2004), 727-749. 2

[Dar93] DARIPA P.: A fast algorithm to solve the Beltrami equation with applications to quasiconformal mappings. Journal of Computational Physics 106, 2 (1993), 355-365. 2

[DMA02] Desbrun M., Meyer M., Alliez P.: Meshes and parameterization: Intrinsic parameterizations of surface meshes. Computer Graphics Forum 21, 3 (2002), 209. 2, 8

[ESG01] Eckstein I., Surazhsky V., Gotsman C.: Texture mapping with hard constraints. In Computer Graphics Forum (2001), vol. 20, Wiley Online Library, pp. 95-104. 2

[FH05] FlOATER M., HoRmann K.: Surface parameterization: a tutorial and survey. Advances In Multiresolution For Geometric Modelling (2005). 1

[Flo97] FloATER M.: Parametrization and smooth approximation of surface triangulations. Computer Aided Geometric Design 14, 3 (1997), 231-250. 2

[Gar] Gardiner. F. P.: Personal communication. 4

[GL00] GARDINER F., LAKIC N.: Quasiconformal Teichmüller theory, vol. 76 of Mathematical Surveys and Monographs. American Mathematical Society, 2000. 2

[Grö30] GRÖTZSCH H.: Ueber die Verzerrung bei nichtkonformen schlichten Abbildungen mehrfach zusammenhängender Bereiche. Leipz. Ber. 82 (1930), 69-80. 2

[GY03] GU X., YAU S.-T.: Global conformal surface parameterization. In Symposium on Geometry Processing (Aire-laVille, Switzerland, 2003), SGP '03, Eurographics Association, pp. $127-137.2$

[HG99] Hormann K., Greiner G.: MIPS: An efficient global parameterization method. Curve and Surface Design: Saint-Malo 2000 (1999), 153-162. 2, 8

[JZDG09] Jin M., ZENG W., Ding N., Gu X.: Computing Fenchel-Nielsen coordinates in Teichmuller shape space. In Shape Modeling International (2009), IEEE, pp. 193-200. 2

[JZLG09] JIN M., ZENG W., LuO F., GU X.: Computing Tëichmuller shape space. Visualization and Computer Graphics 15, 3 (2009), 504-517. 2

[KGG05] Karni Z., Gotsman C., Gortler S.: Freeboundary linear parameterization of $3 \mathrm{~d}$ meshes in the presence of constraints. In Shape Modeling and Applications, 2005 International Conference (2005), IEEE, pp. 266-275. 2

[KMZ10] Kovacs D., MYles A., Zorin D.: Anisotropic quadrangulation. Symposium on Solid and Physical Modeling (2010), 137-146. 6

[KNP07] KÄlberer F., Nieser M., Polthier K.: QuadCover: surface parameterization using branched coverings. Computer Graphics Forum 26, 3 (2007), 375-384. 2

[KSG03] Kraevoy V., Sheffer A., Gotsman C.: Matchmaker: constructing constrained texture maps. In ACM Trans. Graph. (2003), vol. 22, ACM, pp. 326-333. 2

[KSS06] KHAREVYCH L., SPRINGBORN B., SCHRÖDER P.: Discrete conformal mappings via circle patterns. ACM Trans. Graph. 25 (April 2006), 412-438. 2

(c) 2012 The Author(s)

(c) 2012 The Eurographics Association and Blackwell Publishing Ltd.
[Lév01] LÉvY B.: Constrained texture mapping for polygonal meshes. In Proc. Computer graphics and interactive techniques (2001), ACM, pp. 417-424. 2

[LKF12] Lipman Y., Kim V., FUnKHOUSER T.: Simple formulas for quasiconformal plane deformations. ACM Trans. Graph. (2012). to appear. 2

[LPRM02] LÉvy B., Petitjean S., Ray N., Maillot J.: Least squares conformal maps for automatic texture atlas generation. ACM Trans. Graph. 21, 3 (2002), 362-371. 2, 8

[LWT*10] Lui L., Wong T., Thompson P., Chan T., Gu X., YAU S.: Shape-based diffeomorphic registration on hippocampal surfaces using Beltrami holomorphic flow. Med. Image Comput. and Comp.-Assisted Intervention (2010), 323-330. 2

[LZX*08] LiU L., Zhang L., Xu Y., Gotsman C., Gortler S. J.: A local/global approach to mesh parameterization. Computer Graphics Forum 27, 5 (July 2008), 1495-1504. 2, 8

[Rei02] REICH E.: Extremal quasiconformal mappings of the disk. Handbook of Complex Analysis 1 (2002), 75-136. 4

[RLL*06] RAY N., Li W., LÉvy B., ShefFer A., Alliez P.: Periodic global parameterization. ACM Trans. Graph. 25, 4 (2006), 1460-1485. 2

[SdS01] Sheffer A., DE STURler E.: Parameterization of faceted surfaces for meshing using angle-based flattening. Engineering with Computers 17, 3 (2001), 326-337. 2

[SM04] Sharon E., MuMFord D.: 2d-shape analysis using conformal mapping. In Computer Vision and Pattern Recognition (2004), vol. 2, IEEE, pp. II-350. 2

[SSGH01] Sander P., Snyder J., Gortler S., Hoppe H.: Texture mapping progressive meshes. In Proc. Computer graphics and interactive techniques (2001), ACM, pp. 409-416. 2

[SSP08] Springborn B., Schröder P., Pinkall U.: Conformal equivalence of triangle meshes. ACM Trans. Graph. 27 (August 2008), 77:1-77:11. 2, 5, 6, 10

[Tei40] TEICHMÜlLER O.: Extremale quasikonforme Abbildungen und quadratische Differentiale. Preuss. Akad. Math.-Nat., 22 (1940). 2

[Tei43] TEICHMÜLlER O.: Bestimmung der extremalen quasikonformen Abbildungen bei geschlossenen orientierten Riemannschen Flächen. Preuss. Akad. Math.-Nat. 4 (1943). 2

[Tut63] TutTe W.: How to draw a graph. Proc. London Math. Soc 13, 3 (1963), 743-768. 2

[WDC*09] Wang Y., Dai W., Chou Y., Gu X., Chan T., Toga A., ThOMPSON P.: Studying brain morphometry using conformal equivalence class. In Computer Vision, 12th International Conference on (2009), IEEE, pp. 2365-2372. 2

[ZLYG09] ZENG W., LuO F., YAu S., Gu X.: Surface quasiconformal mapping by solving Beltrami equations. Mathematics of Surfaces XIII (2009), 391-408. 2

[ZRS05] ZAYER R., Rossl C., SeIDEl H.: Discrete tensorial quasi-harmonic maps. Proc. Shape Modeling and Applications (2005), 276-285. 2 